\title{
POINT-FINITE BOREL-ADDITIVE FAMILIES ARE OF BOUNDED CLASS
}

\author{
R. W. HANSELL
}

\begin{abstract}
We prove the following theorem, which answers a question originally raised by J. Kaniewski and R. Pol:

THEOREM. If $\mathcal{X}$ is a point-finite family of subsets of a metric space $X$ such that the union of every subfamily is a Borel set of $X$, then there exists a fixed countable ordinal $\alpha$ such that each member of $\mathcal{X}$ is a Borel set of class $\alpha$ in $X$.

The proof is given in the general setting of abstract measurable spaces. An application is made to the study of measurable selectors for compact-valued mappings and to the Borel measurability of graphs.
\end{abstract}

In their joint paper [5], J. Kaniewski and R. Pol raised the question of whether a point-finite Borel-additive family in a metric space has the property that, for some fixed $\alpha<\omega_{1}$, all members of the family are of class $\alpha$ (cf. [5, Question 3, p. 1049], and also [1, Question 5, p. 315] and [4, Problem 10, p. 482]). For disjoint families, D. Preiss [7] has shown that such a bound exists, even in the more general setting of abstract "measurable spaces" (see the paper by W. Fleissner [1] for a short and elegant proof of Preiss's theorem). The purpose of this note is to prove that Preiss's result also holds in the point-finite case, and that this cannot be extended to the case of point-countable families. ${ }^{1}$

Let $X$ be any set, and let $\mathcal{E}$ be a family of subsets of $X$. We define inductively the family $\mathcal{L}_{\alpha}$, for $\alpha<\omega_{1}$, by letting $\mathscr{L}_{0}=\mathcal{L}$, and defining $\mathcal{L}_{\alpha}$ to be the collection of all countable unions or intersections of members of $U\left\{\mathcal{L}_{\beta}: \beta<\alpha\right\}$ according as $\alpha$ is even or odd (treating limit ordinals as even). To avoid trivialities, in this context we will always assume that $\varnothing$ belongs to $\mathcal{L}$. We put, $\sigma(\mathcal{L})=\cup_{\alpha<\omega_{1}} \mathcal{L}_{\alpha}$.

A family $\mathcal{X}$ of subsets of $X$ is said to be $\mathscr{N}$-additive, where $\Re$ is any collection of subsets of $X$, provided the union of every subfamily of $\mathcal{X}$ belongs to $\Re$. The family $\mathcal{X}$ is said to be point-finite if each point of $X$ belongs to at most a finite number of members of $\mathcal{X}$. We will prove the following:

THEOREM 1. If $\mathcal{X}$ is a point-finite $\sigma(\mathcal{L})$-additive family of subsets of a set $X$, then there exists an $\alpha<\omega_{1}$ such that $\mathcal{X} \subset \mathcal{L}_{\alpha}$.

We first prove a lemma, which may be of some independent interest.

Received by the editors October 30, 1980 and, in revised form, February 10, 1981.

1980 Mathematics Subject Classification. Primary 04A15, 28A05, 54H05; Secondary 54C60, 54 C65.

Key words and phrases. Borel-additive family, point-finite family, Borel classifications, measurable selectors.

${ }^{1}$ Professor Roman Pol has recently informed me that his student S. Spahn has also independently proven Theorem 1 of this paper using different methods. The results of this paper were first announced by the author in [3]. 
LeMma 1. Let $S$ be a separable metric space, and suppose $\left\{X_{s}: s \in S\right\}$ is a point-finite $\sigma(\mathcal{L})$-additive family in $X$. Then $\{X s \times\{s\}: s \in S\}$ is $\sigma(\Re)$-additive in $X \times S$ where $\Re=\mathcal{L} \times \mathcal{T}$ and $\mathcal{T}$ is the topology of $S$.

Proof. Since $S$ can be embedded in a compact metric space, we may choose a countable open base for $S,\left\{U_{n}: n \in \omega\right\}$, such that $\operatorname{diam}\left(U_{n}\right) \rightarrow 0$ and each point of $S$ belongs to $U_{n}$ for arbitrarily large values of $n$ (this is possible since $S$ has at most a countable number of isolated points).

Fix $A \subset S$, and put

$$
B=\bigcup\left\{X_{s} \times\{s\}: s \in A\right\} \text { and } M_{n}=\bigcup\left\{X_{s}: s \in U_{n} \cap A\right\} .
$$

Since the countable family $\left\{M_{n}: n \in \omega\right\}$ must range in some $\mathfrak{L}_{\alpha}$, to show that $B \in \sigma(\Re)$ it suffices to show that

$$
B=\bigcap_{m=1}^{\infty} \bigcup_{n=m}^{\infty} M_{n} \times U_{n} .
$$

The inclusion $\subset$ in (1) is clear, since each point of $S$ belongs to a subsequence of $\left\{U_{n}: n \in \omega\right\}$.

Now suppose $\left(x_{0}, s_{0}\right) \in M_{n_{m}} \times U_{n_{m}}$ where $n_{m}>m, m \in \omega$. Since $x_{0} \in M_{n_{m}}$, we can choose $s\left(n_{m}\right) \in U_{n_{m}} \cap A$ such that $x_{0} \in X_{s\left(n_{m}\right)}$. By the point-finiteness of the family $\left\{X_{s}: s \in A\right\}$, there must be infinitely many distinct indices $m$ such that the corresponding points $s\left(n_{m}\right)$ are the same, and we denote this common point by $s_{1}$. Since both $s_{0}$ and $s_{1}$ belong to the same subsequence of $\left\{U_{n_{m}}: m \in \omega\right\}$, and $\operatorname{diam}\left(U_{n}\right) \rightarrow 0$, we have $s_{0}=s_{1}$. It follows that $s_{0} \in A$ and $x_{0} \in X_{s_{0}}$, and so $\left(x_{0}, s_{0}\right) \in B$. That completes the proof of the lemma.

The following lemma is also needed. The proof is a straightforward induction argument and is left to the reader.

Lemma 2. Let $\mathcal{L}$ and $\mathfrak{T}$ be families of subsets of the sets $X$ and $Y$, respectively, and let $\Re=\mathcal{L} \times \mathfrak{R}$. For all $H \subset X \times Y$ and $\beta<\omega_{1}$, if $H \in \Re_{\beta}$, then $H_{y}=\{x$ $\in X:(x, y) \in H\}$ belongs to $\mathfrak{L}_{\beta}$ for every $y \in Y$.

Proof of Theorem 1. Suppose, on the contrary, that $\mathcal{X} \not \mathfrak{L}_{\alpha}$ for every $\alpha<\omega_{1}$. Thus we may choose inductively sets

$$
X_{\alpha} \in \mathcal{X}-\left(\mathfrak{L}_{\alpha} \cup\left\{X_{\beta}: \beta<\alpha\right\}\right)
$$

for every $\alpha<\omega_{1}$. Treating $S=\left\{\alpha: \alpha<\omega_{1}\right\}$ as a subspace of the reals (say), we may apply Lemma 1 to conclude that

$$
H=\bigcup\left\{X_{\alpha} \times\{\alpha\}: \alpha<\omega_{1}\right\} \in \Re_{\beta+1},
$$

for some $\beta<\omega_{1}$. Since $X \times\{\alpha\} \in \Re_{1}$, it follows that

$$
H \cap(X \times\{\alpha\})=X_{\alpha} \times\{\alpha\} \in \Re_{\beta+1},
$$

for each $\alpha<\omega_{1}$. But this implies that, for every $\alpha<\omega_{1}, X_{\alpha} \in \mathfrak{L}_{\beta+1}$ in view of Lemma 2. This contradicts our choice of $X_{\alpha}$ for $\alpha>\beta+1$. The theorem is thus proved. 
In [5] it was observed that if Theorem 1 were true, then the following would be a corollary to the proof of Theorem 2 of that paper:

COROllary 1. Let $X$ be an absolutely analytic metric space and $Y$ an arbitrary metric space. If $F: X \rightarrow Y$ is a compact-valued (multi-) map such that $F^{-1}(U)$ $(=\{x \in X: F(x) \cap U \neq \varnothing\})$ is a Borel set in $X$ for every open set $U \subset Y$, then there is a fixed ordinal $\alpha<\omega_{1}$ such that $F^{-1}(U)$ is of additive class $\alpha$ in $X$ for all open $U \subset Y$. Moreover, $F$ admits a selector of class $\alpha$.

Corollary 2. Let $X$ and $Y$ be metric spaces. If $F: X \rightarrow Y$ is a compact-valued map such that $F^{-1}(U)$ is a Borel set in $X$ whenever $U$ is open in $Y$, then

$$
\operatorname{Gr}(F)=\{(x, y) \in X \times Y: y \in F(x)\}
$$

is a Borel set in $X \times Y$.

Proof. For a given metric on $Y$ and for a fixed $n \in \omega$, let $\mathscr{Q}_{n}$ be a locally-finite open cover of $Y$ by sets of diameter $<1 / n$. The assumptions on $F$ imply that the family $\left\{F^{-1}(U): U \in \mathcal{Q}_{n}\right\}$ is point-finite and Borel-additive, and so there exists some $\alpha_{n}<\omega_{1}$ such that each member of the family is of additive class $\alpha_{n}$ in $X$ by Theorem 1. The assumptions on $\mathscr{Q}_{n}$ now imply that the set

$$
B_{n}=\bigcup\left\{F^{-1}(U) \times U: U \in \mathscr{Q}_{n}\right\}
$$

is locally of additive class $\alpha_{n}$ in $X \times Y$, and thus is itself of this class in $X \times Y$ by the theorem of Montgomery [6, p. 358].

Doing this for each $n \in \omega$, we proceed to show that $\operatorname{Gr}(F)=\cap_{n \in \omega} B_{n}$. Thus suppose for each $n \in \omega$ the point $(x, y)$ belongs to $F^{-1}\left(U_{n}\right) \times U_{n}$ for some $U_{n} \in \mathcal{Q}_{n}$. Since $\left\{U_{n}: n \in \omega\right\}$ will then be a local base for $y$ and $F(x) \cap U_{n} \neq \varnothing$ for each $n$, it follows that $y \in F(x)$ (since $F(x)$ is closed), and so we have that (x, $y) \in \operatorname{Gr}(F)$. Since the reverse inclusion is obvious, the conclusion of the corollary follows.

EXAMPLE. In the space $\mathbf{R}$ of real numbers, there exists a point-countable Borel-additive family which is not contained in $\Sigma_{\alpha}$ for any $\alpha<\omega_{1}$ (here $\Sigma_{\alpha}$ denotes the family of sets of additive class $\alpha$ in $\mathbf{R}$ ).

Construction. There exists a disjoint family $\left\{B_{\alpha}\right\}_{\alpha<\omega_{1}}$ of Borel sets of $\mathbf{R}$ of unbounded class with $\mathbf{R}=\cup_{\alpha<\omega_{1}} B_{\alpha}$ (see, e.g., [6, p. 484]). Let $C_{\alpha}=\cup_{\beta<\alpha} B_{\beta}$ for each $\alpha<\omega_{1}$. Since $B_{\alpha}=C_{\alpha}-\cup_{\beta<\alpha} C_{\beta}$ for each $\alpha<\omega_{1}$, it is clear that $\left\{C_{\alpha}\right\}_{\alpha<\omega_{1}}$ is an ascending transfinite sequence of Borel sets of $\mathbf{R}$ of unbounded class. It thus follows that the descending transfinite sequence $\left\{\mathbf{R}-C_{\alpha}\right\}_{\alpha<\omega_{1}}$ is point-countable, Borel-additive and $\varnothing \Sigma_{\alpha}$ for every $\alpha<\omega_{1}$.

The following question arises naturally in relation to Theorem 1.

Question. Given $X$ and $\mathcal{L}$ as above, if $\mathcal{X}$ is a point-finite $\sigma(\mathfrak{L})$-additive family in $X$, does there exist an $\alpha<\omega_{1}$ such that $\mathcal{X}$ is $\mathfrak{L}_{\alpha}$-additive?

This is a variation of a question first posed by W. Fleissner in [1] (see also [4, Problem 15] and [3]). Some partial affirmative answers are known, but we know of no partial negative answers. For instance, if $\mathcal{L}$ is the family of closed sets of a topological space $X$ and $\mathcal{X}$ is (in addition) " $\sigma$-discretely decomposable", then the question is answered in the affirmative (see [1], [3]); in particular, this is the case if 
either $X$ is an absolutely analytic metric space [5], or $X$ is metric and "Proposition P" holds [1], or if $X$ is "of weak character below $C$ " and the "Product Measure Extension Axiom" is assumed [2]. Most recently, the author has shown that the question (as stated) can be answered in the affirmative if one assumes the existence of an uncountable separable metric space all subsets of which are Borel (hence under MA $+\neg \mathrm{CH}$ ). However, the complete answer remains open, even for the special case when $\mathcal{X}$ is disjoint.

\section{REFERENCES}

1. W. G. Fleissner, An axiom for nonseparable Borel theory, Trans. Amer. Math. Soc. 251 (1979), 309-328.

2. W. G. Fleissner, R. W. Hansell and H. J. K. Junnila, PMEA implies Propasition P, General Topology Appl. (to appear).

3. R. W. Hansell, Borel-additive families and Borel maps in metric spaces, General Topology and Modern Analysis, edited by L. F. McAuley and M. M. Rao, Academic Press, New York, 1981.

4. C. A. Rogers (Editor), Analytic sets, Academic Press, New York, 1980.

5. J. Kaniewski and R. Pol, Borel-measurable selectors for compact-valued mappings in the nonseparable case, Bull. Acad. Polon. Sci. Sér. Sci. Math. Astronom. Phys. 23 (1975), 1043-1050.

6. K. Kuratowski, Topology, Vol. 1, Academic Press, New York; PWN, Warsaw, 1966.

7. D. Preiss, Completely additive disjoint systems of Baire sets is of bounded class, Comment. Math. Univ. Carolinae 15 (1972), 341-344.

Department of Mathematics, University of ConNecticut, Storrs, CONNecticut 06268 